\title{
Polarization-sensitive optical coherence tomography imaging of the anterior mouse eye
}

Bernhard Baumann

Marco Augustin

Antonia Lichtenegger

Danielle J. Harper

Martina Muck

Pablo Eugui

Andreas Wartak

Michael Pircher

Christoph K. Hitzenberger 


\title{
Polarization-sensitive optical coherence tomography imaging of the anterior mouse eye
}

\author{
Bernhard Baumann, ${ }^{\mathrm{a}, \star}$ Marco Augustin, ${ }^{\mathrm{a}}$ Antonia Lichtenegger, ${ }^{\mathrm{a}}$ Danielle J. Harper, ${ }^{\mathrm{a}}$ Martina Muck, ${ }^{\mathrm{a}, \mathrm{b}}$ \\ Pablo Eugui, ${ }^{a}$ Andreas Wartak, ${ }^{a}$ Michael Pircher, ${ }^{a}$ and Christoph K. Hitzenberger ${ }^{\mathrm{a}}$ \\ ${ }^{a}$ Medical University of Vienna, Center for Medical Physics and Biomedical Engineering, Vienna, Austria \\ ${ }^{b}$ Medical University and General Hospital of Vienna, Institute of Neurology, Vienna, Austria
}

\begin{abstract}
Polarization-sensitive optical coherence tomography (PS-OCT) enables noninvasive, high-resolution imaging of tissue polarization properties. In the anterior segments of human eyes, PS-OCT allows the visualization of birefringent and depolarizing structures. We present the use of PS-OCT for imaging the murine anterior eye. Using a spectral domain PS-OCT setup operating in the 840-nm regime, we performed in vivo volumetric imaging in anesthetized C57BL/6 mice. The polarization properties of murine anterior eye structures largely replicated those known from human PS-OCT imagery, suggesting that the mouse eye may also serve as a model system under polarization contrast. However, dissimilarities were found in the depolarizing structure of the iris which, as we confirmed in postmortem histological sections, were caused by anatomical differences between both species. In addition to the imaging of tissues in the anterior chamber and the iridocorneal angle, we demonstrate longitudinal PS-OCT imaging of the murine anterior segment during mydriasis as well as birefringence imaging of corneal pathology in an aged mouse. ๑ The Authors. Published by SPIE under a Creative Commons Attribution 3.0 Unported License. Distribution or reproduction of this work in whole or in part requires full attribution of the original publication, including its DOI. [DOI: 10 1117/1.JBO.23.8.086005]
\end{abstract}

Keywords: optical coherence tomography; polarization-sensitive devices; mouse; ophthalmology; birefringence; depolarization; cataract.

Paper 180330R received Jun. 5, 2018; accepted for publication Aug. 14, 2018; published online Aug. 30, 2018.

\section{Introduction}

Polarization-sensitive optical coherence tomography (PS-OCT) combines the high-resolution imaging capabilities of OCT with polarization contrast. ${ }^{1,2}$ Polarization of light thereby may provide additional image contrast based on inherent polarization properties related to the textural organization of tissues, such that structures can be distinguished for example based on their birefringent characteristics. PS-OCT has found widespread applications in many biomedical fields including imaging of dental, neural, osseous, and intravascular structures. ${ }^{1,2}$ The most prominent field of application of PS-OCT, however, has been ophthalmology. ${ }^{3}$ In the human eye, PS-OCT can be used to distinguish: (a) fibrous tissues exhibiting birefringence including the retinal nerve fiber layer, sclera, cornea, and tendons of extraocular muscles; ${ }^{4-13}$ (b) pigmented structures exhibiting polarization scrambling including the retinal pigment epithelium, the choroid, and the pigment epithelium of the iris, ${ }^{5,14}$ and (c) polarization preserving tissues including the iridal stroma, conjunctive tissue, and most of the neurosensory retina such as the photoreceptor layer. ${ }^{5,15}$ PS-OCT has proven particularly useful for imaging and quantitative assessment of retinal pathology in age-related macular degeneration, glaucoma, central serous retinopathy, and diabetic retinopathy. ${ }^{8,16-21}$

In images of the anterior eye segment, PS-OCT provides additional contrast for collagenous, birefringent tissues such as the cornea, sclera, and tendons, as well as for the trabecular meshwork. ${ }^{9,12,15}$ The diagnostic potential of birefringence imaging by PS-OCT has been exploited in keratoconus patients. ${ }^{9,22}$

*Address all correspondence to: Bernhard Baumann, E-mail: bernhard .baumann@meduniwien.ac.at
As alterations in corneal microstructure can lead to changes of the birefringent characteristics, PS-OCT was also proposed for imaging such changes during corneal crosslinking therapy. ${ }^{12,23}$ Following trabeculectomy - a surgical procedure for intraocular pressure (IOP) relieve in glaucoma patients - the evolution of filtering blebs was monitored by PS-OCT. ${ }^{12,24-27}$ Finally, PSOCT was also used to investigate birefringence characteristics of the sclera. ${ }^{13}$

For advancing our knowledge and understanding of eye development, its physiology in health and disease, and for the development of new therapeutics, rodent models have been playing an essential role. ${ }^{28,29}$ The gold standard for such preclinical investigations has been histology. Histology enables the assessment of structural details with microscopic resolution and molecular specificity. However, it also requires enucleation of the eye, and therefore, does not permit longitudinal studies in the same animal. OCT - among other modalities - has been proposed as a noninvasive alternative to histology providing in vivo imaging capabilities with micrometer scale resolution and in real time. OCT has been applied for imaging the anterior murine eye in models of several ophthalmic diseases including a model of Axenfeld-Rieger malformations of the anterior segment related to PITX2 mutation, ${ }^{30}$ a DBA/2J model of glaucoma, ${ }^{31}$ a mouse model of glaucoma overexpressing bone morphogenetic protein $2,{ }^{32}$ mouse models of elevated $\mathrm{IOP}^{33,34}$ mice deficient in secreted protein acidic and rich in cysteine, ${ }^{35}$ and phenotypical investigations of genetic components of corneal and stromal thickness relevant for keratoconus. ${ }^{36}$

Even though standard (i.e., non-PS) OCT has obviously proven useful for imaging the anterior segment in preclinical studies, PS-OCT studies of the murine eye have thus far been limited to the posterior eye segment. ${ }^{37-40}$ In this paper, 
we aim to investigate the polarization properties of the healthy murine eye and demonstrate the potential of PS-OCT as a tool for imaging structural changes in the anterior mouse eye with enhanced contrast.

\section{Materials and Methods}

\subsection{Animals}

Pigmented C57BL/6 mice were purchased from the breeding facility at the Medical University of Vienna. The mice were fed a standard diet and were kept under controlled lighting conditions (12-h light, 12-h dark). For the OCT imaging experiments, mice ( $N=5$, age: 20 to 21 months) were anesthetized using ketamine (Ketasol, aniMedica $\mathrm{GmbH}$, Germany; $100 \mathrm{mg} / \mathrm{kg}$ body weight; intraperitoneal) and xylazine (Rompun, Bayer Austria $\mathrm{GmbH}$, Austria; $5 \mathrm{mg} / \mathrm{kg}$ body weight; intraperitoneal). One mouse (age 20 months) which presented an irregular cornea was imaged as a pathologic example, and one mouse (age 21 months) was used for a longitudinal investigation of the anterior eye during mydriasis. Anesthesia was required for immobilizing the animals during PS-OCT imaging. For imaging the murine lens in some animals, the pupils were dilated using phenylephrine $(2.5 \%$; topical) and tropicamide (Mydriaticum, Agepha Pharmaceuticals, Austria; topical). Under anesthesia, mice were placed in an adjustable mount in order to align the eye position with respect to the measurement beam and kept warm using heat pads. Artificial tear drops (Oculotect; Thea Pharma GmbH, Austria) were repeatedly applied to keep the cornea moist. All experiments were performed in accordance with the ARVO Statement for the Use of Animals in Ophthalmic and Vision Research and under a protocol approved by the ethics committee at Medical University of Vienna and the Austrian Federal Ministry of Science, Research and Economy (protocol number GZ 66.009/0131-V/3b/2018).

\subsection{Polarization-Sensitive Optical Coherence Tomography}

A sketch of the spectral domain PS-OCT system used for imaging the murine anterior segment is shown in Fig. 1. Detailed specifications of the system can be found elsewhere, ${ }^{41}$ hence only a brief description is provided here. A polarization-maintaining (PM) fiber-based Michelson interferometer was illuminated by a superluminescent diode with a central wavelength of $837 \mathrm{~nm}$ and a full-width-at-half-maximum bandwidth of $52 \mathrm{~nm}$. The incident light was linearly polarized, launched into the slow axis channel of the PM fiber system, and split into sample and reference arms. A quarter wave plate (QWP, at $45 \mathrm{deg}$ ) then provided circularly polarized light for sample illumination. After passing a pair of galvanometer scanners, a 4:5 telescope relayed the pivot point of the fast scanning mirror into the back focal plane of the $10 \times$ objective lens (Olympus E A10). The setup provided a transverse resolution of $\sim 10 \mu \mathrm{m}$ in the focal plane and an axial resolution of $7.6 \mu \mathrm{m}$ in air. The reference arm featured another QWP (at $22.5 \mathrm{deg}$ ) and glass blocks for dispersion balancing. The interfered light from sample and reference arm was split into two orthogonal components by a polarizing beam splitter. Each polarization component was then detected by a spectrometer unit comprising a collimator $(f=100 \mathrm{~mm})$, a transmission grating (1200 lines $/ \mathrm{mm}$ ), an achromatic doublet $(f=200 \mathrm{~mm})$, and a high-speed line scan camera (Basler sprint) operating at $70 \mathrm{kHz}$ line rate using 2048 pixels per spectrum corresponding to a frame rate of $\sim 68 \mathrm{~B}$-scans per second. A real-time display providing B-scan images at 28 frames per second and a continuously updating OCT z-projection image enabled speedy alignment of the mouse eye with respect to the beam. Data sets containing $1024(x) \times 200(y) \times$ $2048(k)$ pixels per polarization channel covering up to $3.5 \mathrm{~mm}(x) \times 3.5 \mathrm{~mm}(y) \times 2.7 \mathrm{~mm}(z$, optical distance $)$ were acquired in $\sim 3 \mathrm{~s}$.

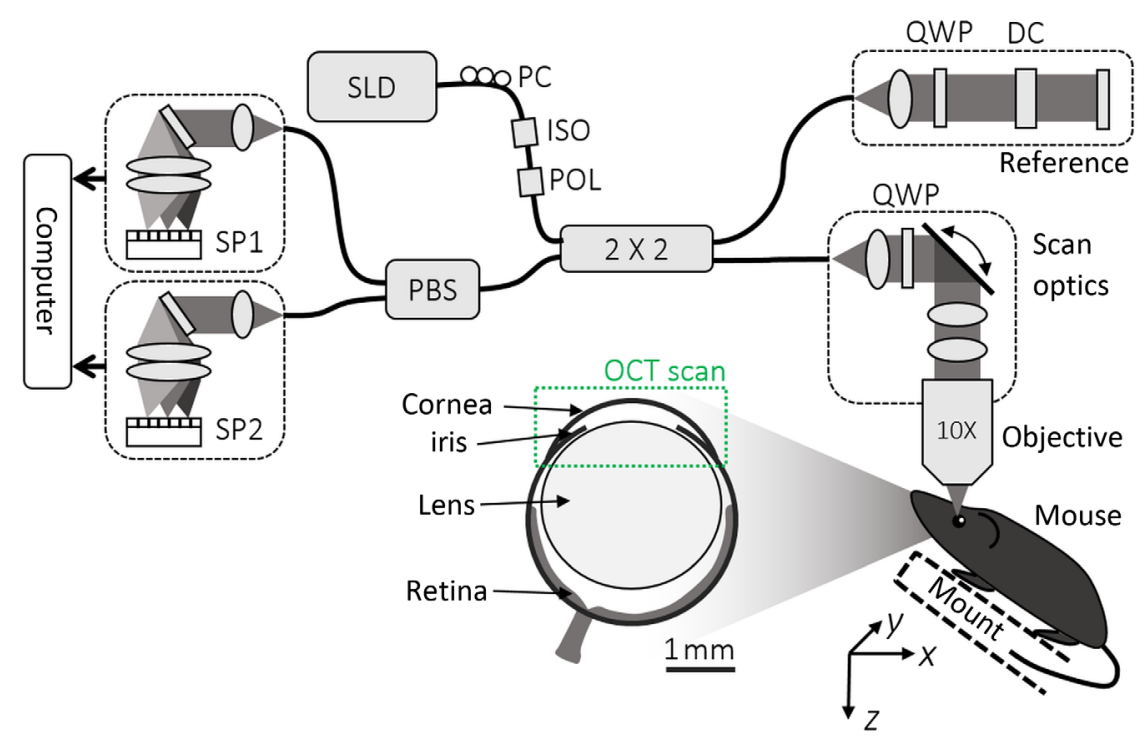

Fig. 1 Scheme of the spectral domain PS-OCT prototype and imaging setup. A PM-fiber-based Michelson interferometer and a pair of high-speed spectrometers are the core of the system. The beam in the sample arm scans the mouse eye via a pair of galvanometer steered mirrors. The mouse resided on a heated stage during imaging. The location of the OCT scan is shown in green color along with an overview of the anatomical structure. SLD, superluminescent diode; PC, polarization control; ISO, optical isolator; POL, polarizer; $2 \times 2$, fiber-optic 50/50 beam splitter; QWP, quarter wave plate; DC, dispersion compensation; PBS, polarizing beam splitter; and SP1/SP2, spectrometers. 


\subsection{PS-OCT Image Processing}

PS-OCT images were computed from the spectral data by first performing standard spectral domain OCT processing including background removal, resampling to $k$-space, numerical dispersion compensation, zero-padding, and Fourier transformation. ${ }^{42}$ Compared to results from earlier imaging sessions in the rodent retina ${ }^{37}$ and in the human brain, ${ }^{43}$ OCT images of the murine anterior segment include structures with greatly varying reflectivity-from the corneal epithelium with signals close to the noise floor to the densely pigmented iris pigment epithelium with signals $>40 \mathrm{~dB}$ above the average noise level. Since the strong backscattering signals were accompanied by $\sim 25 \mathrm{~dB}$ weaker ghost signals caused by distant side lobes of the point spread function, an additional spectral shaping step was implemented. ${ }^{44}$ For this purpose, all spectra of each B-scan acquisition were divided by their average spectrum, which had already served as the background spectrum for background removal. Then each 2048-pixel wide spectrum was multiplied by a Gaussian function with a $1 / \mathrm{e}^{2}$ width of 2200 pixels and by a normalization factor, namely the energy of the background spectrum divided by the energy of the Gaussian shaping function.

After Fourier transforming the preprocessed spectra, the complex data from the two polarization channels were axially aligned in order to compensate for the path length difference caused by the difference in linear polarization mode dispersion of the two orthogonally polarized PM fiber modes. ${ }^{41}$ Finally, PS-OCT images displaying sample reflectivity, phase retardation, and birefringent axis orientation were computed from the amplitude and phase data. ${ }^{6}$ Further, images of the degree of polarization uniformity (DOPU) $)^{45,46}$ were calculated as described in the next section. PS-OCT images were displayed as cross-sectional B-scans and volume renderings using gray and color scales. ${ }^{47}$ In order to gate out regions with low reflectivity, which did not provide meaningful polarization data, pixels whose reflectivity did not exceed the mean noise level by $6 \mathrm{~dB}$ (4 dB for the depolarization analysis in Sec. 2.4) were displayed in light gray in the PS-OCT images. B-scan images with increased SNR were computed by averaging the complex OCT data of several frames repeated at the same $y$-scan position. ${ }^{48}$

\subsection{Depolarization Imaging Using a Simplified Noise Correction for DOPU}

Depolarization of light is caused by several ocular tissues, in particular by those containing melanin pigments. ${ }^{49}$ In order to analyze depolarization in PS-OCT images, the DOPU has emerged as an appropriate and handy approach. DOPU essentially corresponds to a normalized measure of the length of an ensemble of spatially and/or temporally averaged Stokes vectors

$\mathrm{DOPU}=\frac{\sqrt{\bar{Q}^{2}+\bar{U}^{2}+\bar{V}^{2}}}{\bar{I}}$

where $I, Q, U$, and $V$ denote the Stokes vector elements and the overbars indicate an ensemble average. In polarization maintaining tissue, the Stokes vectors within the averaging kernel will be more or less parallel, thus DOPU will be close to unity. In contrast, depolarizing tissue produces Stokes vectors pointing in dissimilar directions, which results in DOPU values smaller than one. As greater polarization scrambling corresponds to a larger solid angle subtended by the Stokes vectors in
Poincaré space, DOPU decreases as depolarization increases. It is important to note that DOPU also depends on the input polarization state. ${ }^{50}$ The most pronounced depolarization effects can be observed for circularly polarized light, which is used for sample illumination here. A variety of different algorithms have been developed to perform DOPU analysis including two- and three-dimensional (3-D) kernels as well as temporal Stokes vector averaging. $41,45,51,52$

One limitation of DOPU is its inaccuracy in case of pixels with low signal-to-noise ratio (SNR). Makita et al. ${ }^{46}$ recently proposed an approach to DOPU, which eliminates the noise bias for the first two Stokes vector elements before averaging, viz. $I^{\prime}=I-\left(\overline{\left|n_{H}\right|^{2}}+\overline{\left|n_{V}\right|^{2}}\right)$ and $Q^{\prime}=Q-\left(\overline{\left|n_{H}\right|^{2}}-\overline{\left|n_{V}\right|^{2}}\right)$, where the primed elements are the noise-error-corrected Stokes parameters and $\overline{\left|n_{p}\right|^{2}}$ is the noise power measured in the $p$ 'th polarization channel. The noise correction improves DOPU measurements in particular for weak signals close to the noise floor (SNR $<10 \mathrm{~dB}$ in reflectivity images). ${ }^{46}$

Now, as in many PS-OCT designs, the optical and electronical layout of the two polarization detection channels is identical, we may assume that $\overline{\left|n_{H}\right|^{2}} \approx \overline{\left|n_{V}\right|^{2}}$. As a consequence, $Q^{\prime} \approx Q$ such that only $I^{\prime}$ is left to be corrected for the additive noise error. By plugging the expression for $I^{\prime}$ into Eq. (1) and using the relation $\overline{\mathrm{SNR}}=\bar{I} /\left(\overline{\left|n_{H}\right|^{2}}+\overline{\left|n_{V}\right|^{2}}\right)$ for the SNR of the PS-OCT reflectivity signal, the expression for the noisecorrected quantity DOPU' simplifies to read

$\mathrm{DOPU}^{\prime}=\frac{\mathrm{DOPU}}{1-\overline{\mathrm{SNR}}^{-1}}$.

Since usually SNR $>1$ in pixels containing meaningful PSOCT data exceeding the noise floor, DOPU' will in general be greater than DOPU. In other words, the DOPU observed without noise correction will be lower than the noise-corrected DOPU', unless the SNR is high. In Fig. 2, we show a comparison of Stokes vector images and DOPU [kernel size: $9(x) \times 5(z)$ pixels] for the original DOPU approach [Fig. 2(a)], ${ }^{45,53}$ Makita's modification [Fig. 2(b)], ${ }^{46}$ and for the simplified approach presented in this paper [Fig. 2(c)]. For the Stokes vector images, the linear magnitudes of Stokes vector elements $Q^{(\prime)}, U$, and $V$ were fed into the R, G, and B channel of an RGB image and the logarithmically scaled $I^{(\prime)}$ was used for the alpha channel. In the DOPU images, pixels with low reflectivity were displayed in gray as described in the previous section. Aside from the more pronounced noise floor intensity in Fig. 2(a), the Stokes vector images look virtually identical for the three approaches, while obvious differences can be observed in the corresponding DOPU images. The polarization preserving or depolarizing characteristics strongly differ for pixels with low SNR (e.g., in deeper regions of the lens) but exhibit a rather similar appearance for pixels with high SNR (e.g., in the lens capsule). Depolarization in the melanin-laden iris leads to a pronounced local variation of the Stokes vectors which is visible as color changes between neighboring speckles in the magnified view of Fig. 2(c) shown in Fig. 2(d). In contrast, the RGB representation of the Stokes vectors in the lens [Fig. 2(e)] appears more uniformly red and orange, indicating a dominant $Q$ component of backscattered light. The second Stokes vector element $Q$ represents the linearly polarized light component, which would be expected to be detected for light scattered from rather polarization preserving tissue after double pass of the QWP in the sample arm. Consequently, the observed DOPU values are low for 
(a)

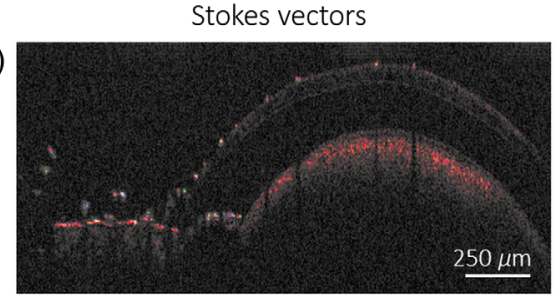

(b)

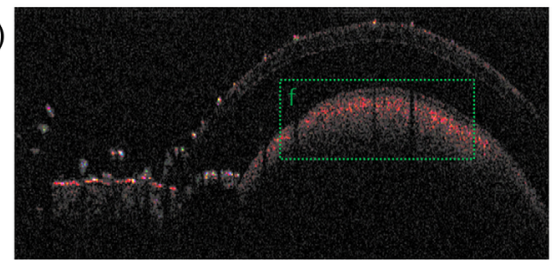

(c)

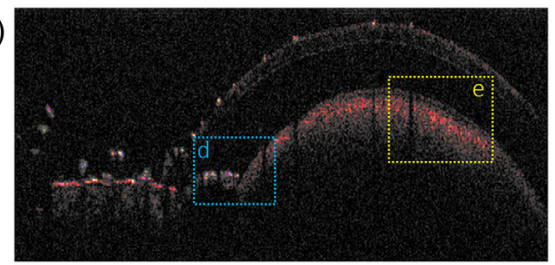

DOPU
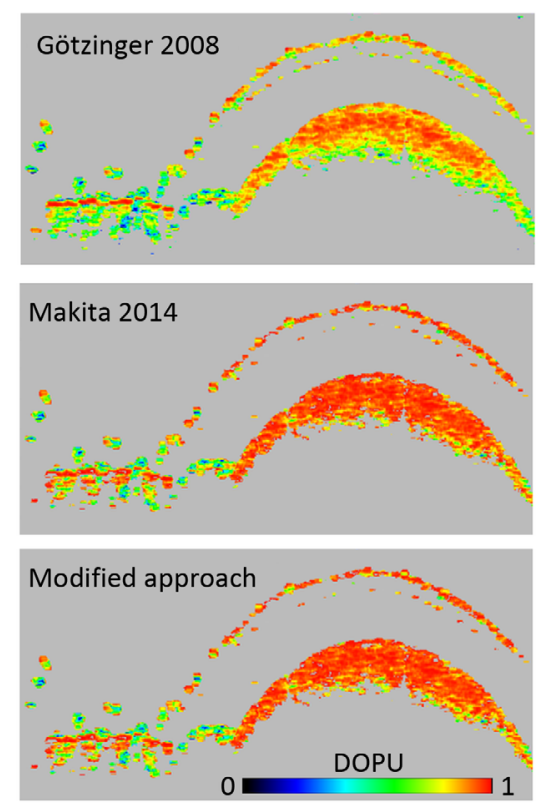

(d)
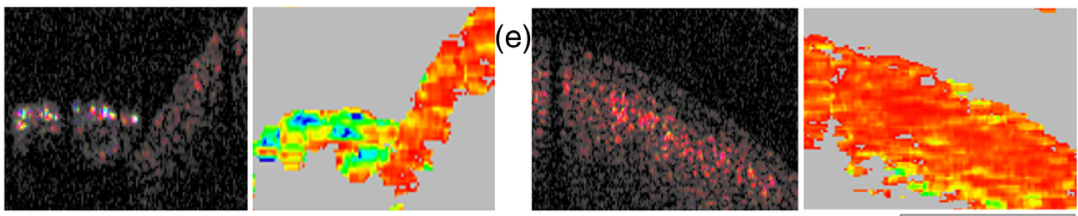

(f)
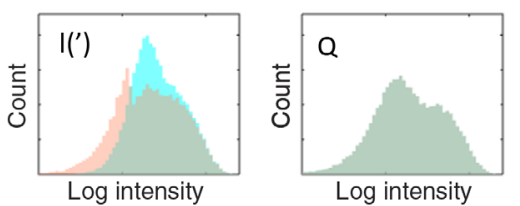

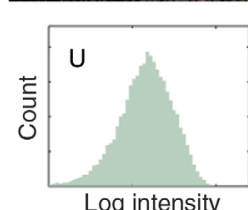

Log intensity

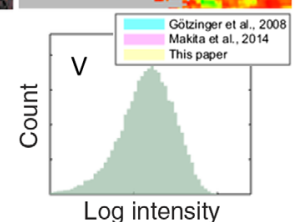

Log intensity

Fig. 2 Stokes vector and DOPU imaging. (a)-(c) Stokes vector images (left) and corresponding DOPU images (right) are shown for (a) the original DOPU approach, ${ }^{45}$ (b) the noise-corrected approach, ${ }^{46}$ and (c) the approach correcting only the noise error of $I$ presented in this paper. For the Stokes vector images, the elements $[Q, U, V]$ are represented in RGB, where $\log [I]$ was used for the alpha channel. In the DOPU images, pixels with subthreshold reflectivity are displayed in gray. (d) and (e) Zoomed views of (d) the iris and (e) lens show depolarization and polarization-preserving characteristics in Stokes vector and DOPU images. (f) Histograms of the Stokes vector element distributions of the B-scans (a)(c) within the range indicated in (b) exhibit no remarkable differences except for the distribution of $I^{(\prime)}$, which is similarly reduced toward lower values for the two noise-error-corrected approaches. The overlap of magenta and yellow histograms for the latter two approaches is apparent as an orange-reddish color. Overlaps of all three histograms produce a common, gray histogram.

Fig. 2(d) and high for Fig. 2(e), respectively. Histograms of the four Stokes vector elements computed for the image region of Figs. 2(a)-2(c) including the lens are shown in Fig. 2(f). Here the distribution of $I^{(\prime)}$ is skewed toward higher values for the original DOPU approach as compared to the noise-corrected approaches which, in turn, appear similar. For the magnitudes of Stokes vector elements $Q, U$, and $V$, the distributions overlap tightly and no differences can be observed. For the DOPU images presented in the results section below, the modified DOPU approach using a rectangular kernel spanning $12(x) \times 5(z)$ pixels was used.

\subsection{Histology Protocol}

For histological correlation, two mice were sacrificed by overdosing with sodium pentobarbital. Mouse eyes were enucleated postmortem after in vivo OCT imaging and fixed in $4 \%$ paraformaldehyde at $4^{\circ} \mathrm{C}$ overnight. Then the eyes were rinsed in phosphate-buffered saline, dehydrated with a series of ascending ethanol concentrations, and embedded in paraffin. Sections of 4- $\mu \mathrm{m}$ thickness were created using a rotary microtome and subsequently stained with hematoxylin and eosin (H\&E). Sections were digitized using the NanoZoomer slide scanner (C9600-12, Hamamatsu).

\section{Results}

\subsection{PS-OCT of the Anterior Eye Segment in C57BL/6 Mice}

PS-OCT imaging was performed in the anterior eye segments of C57BL/6 mice. PS-OCT tomograms of a frontal scan and of the chamber angle of a 20-month-old mouse are shown in Fig. 3. Similar to OCT scans of the human eye, the cornea is rather transparent at $840 \mathrm{~nm}$ and hyposcattering in the reflectivity image [Fig. 3(a)], whereas a strong signal can be observed in 

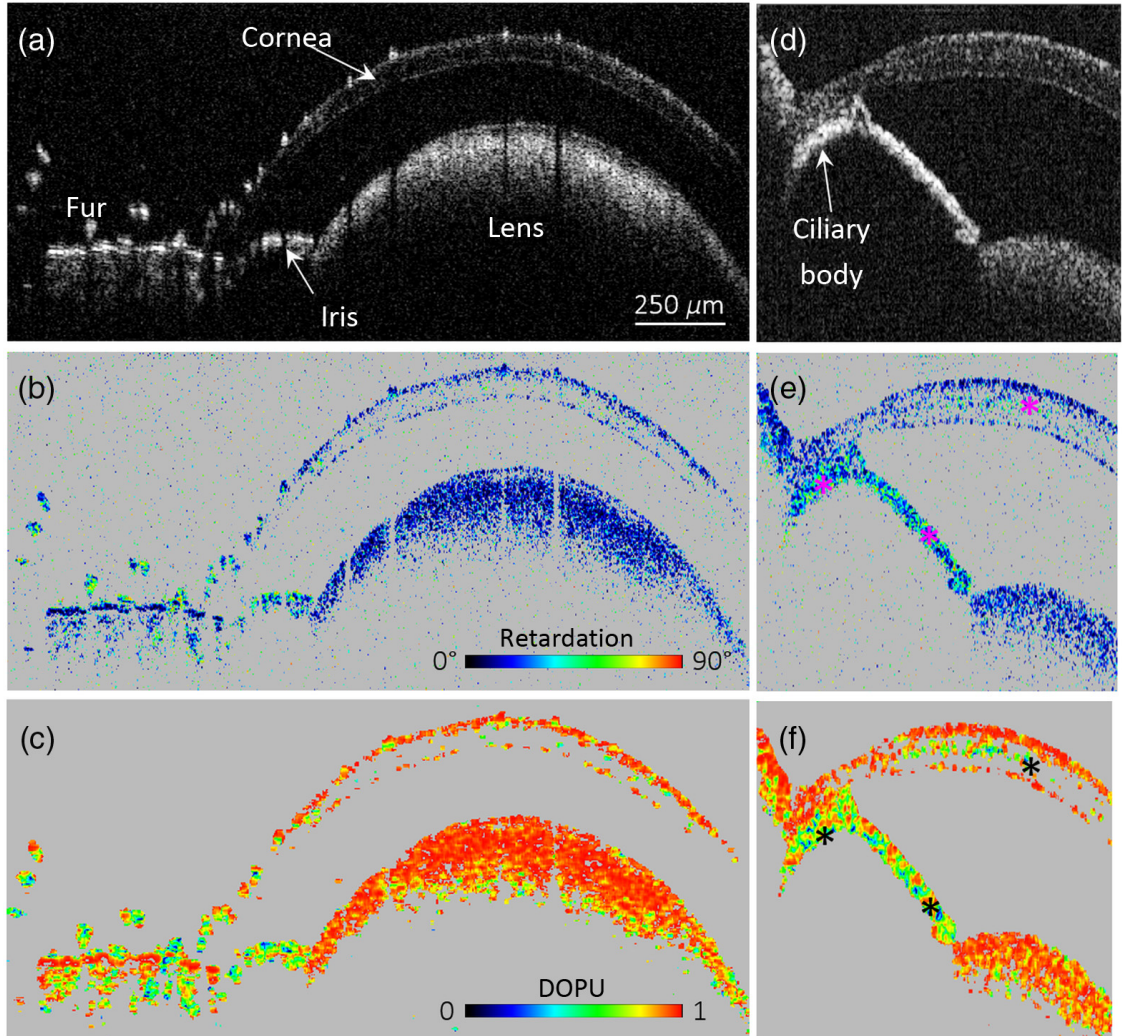

(g)

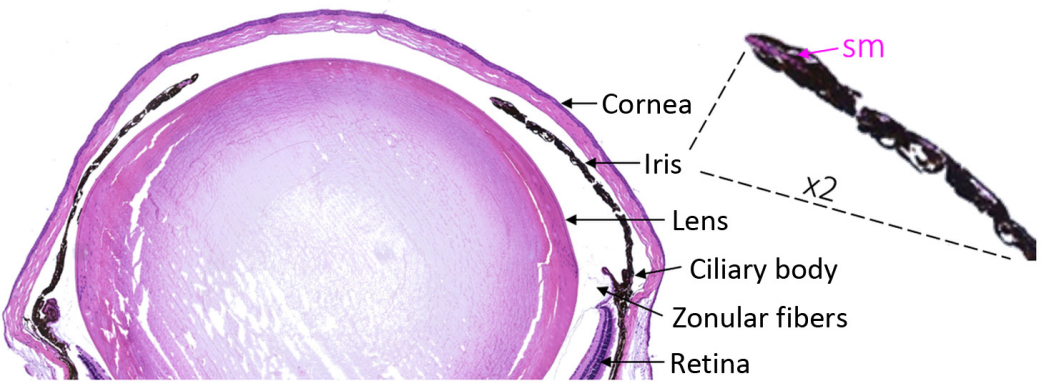

Fig. 3 PS-OCT imaging of the murine anterior segment. (a)-(c) Frontal B-scans of anterior segment in a C57BL/6 mouse. (d)-(f) PS-OCT B-scan images of iridocorneal angle. In panels (a)-(f), the top row shows reflectivity B-scans, the middle row shows the corresponding PS-OCT retardation images, and the bottom row reveals polarization scrambling structures marked by black asterisks in the DOPU images. In panel $(\mathrm{g})$, an H\&E stained histologic section of the anterior mouse eye is shown. Anatomical structures are referenced for comparison to the OCT imagery above. Tissue structures such as iris and ciliary body, which contain the natural chromophore melanin, stand out in brown color in the bright field micrograph. A magnified view of the iris (with sphincter muscle sm) is shown on the right.

the iris. Also the lens provides an intense backscatter signal, which can be attributed to the cataract observed in the aged mouse eye as earlier investigations have shown that normal C57BL/6 mice may develop cataract at older age. ${ }^{54}$ Note that the focal plane was placed approximately at the iris and that due to light attenuation by the iridal melanin, no OCT signals can be observed from structures located posterior to the iris. In the PS-OCT retardation B-scan [Fig. 3(b)], most structures appear uniformly blue with low retardation, only the darkly pigmented iris and fur scramble the polarization and exhibit strongly varying retardation values. A similar picture is seen in the DOPU image in Fig. 3(c). Here most tissues have DOPU values close to unity, except for the iris and the fur whose DOPU values are much lower owing to their pigmentation.

PS-OCT images of the iridocorneal angle are shown in Figs. 3(d)-3(f). These images also provide access to the ciliary body which, similar to the iris, appears hyperscattering in the reflectivity image [Fig. 3(d)] and depolarizing in the retardation and DOPU images, respectively [Figs. 3(e) and 3(f)]. As the focal plane of the objective was placed closer to the cornea for this acquisition, the depolarizing behavior of the corneal stroma can be observed in Figs. 3(e) and 3(f) as well (see asterisks).

In order to compare the PS-OCT data to the gold standard, we show an H\&E stained histological section of a 20-month-old C57BL/6 mouse in Fig. 3(g). The melanin pigments within tissue structures in the uvea-namely iris, ciliary body, and choroid-appear intrinsically dark brown. The magnified view on the panel's right side shows that the pigments are distributed all across the murine iris - unlike the human iris, which only comprises melanin in a thin epithelial layer while its stroma is pigment-free. 

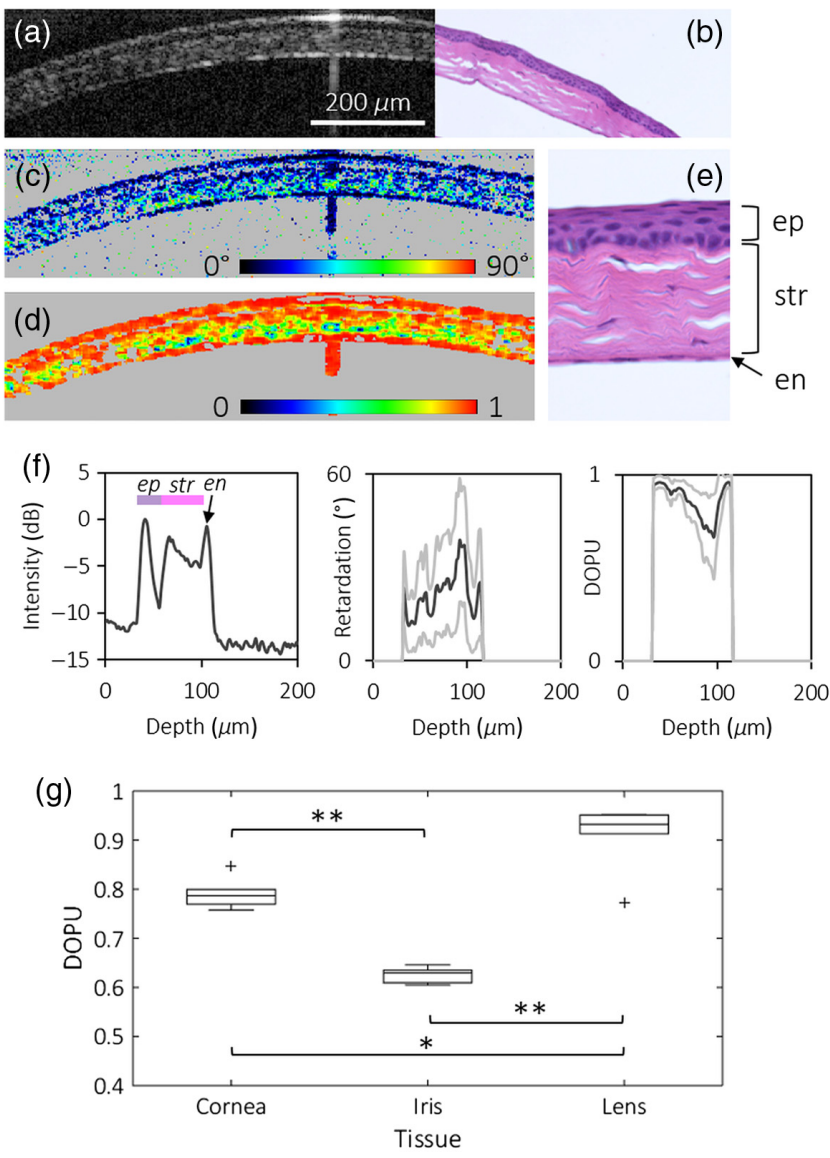

Fig. 4 PS-OCT imaging of the murine cornea and comparative histology. (a) Reflectivity B-scan showing the layered structure of the cornea of a C57BL/6 mouse, (b) comparative histology, (c) retardation Bscan revealing strongly varying retardation in the corneal stroma, and (d) DOPU B-scan. High DOPU values (red) can be observed in the epi- and endothelial shells, while polarization states are rather scrambled in the stroma. (e) High-magnification view of a histological section of the cornea. Labels: epithelium, ep; stroma, str; and endothelium, en. (f) Average axial reflectivity $\left(20 * \log _{10}\right.$ [intensity]), retardation, and DOPU profiles in the mouse cornea. The black and gray plots denote mean \pm standard deviation of values measured at each depth. (g) Box plots showing DOPU measurements in six C57BL/6 mouse eyes. Outliers are plotted as "+" symbols. Statistically significant differences found by a U-test are indicated by ** for $p<0.01$ and by * for $p<0.05$, respectively.

A more detailed view of the polarization characteristic of the murine cornea is provided in Fig. 4. For these images, the cornea overlapped with the system's focal plane and four images were averaged to enhance the SNR and improve the visualization of the corneal stroma. Similar to the human cornea, ${ }^{55}$ the reflectivity is weaker in the corneal epithelium than in the stroma [Fig. 4(a)]. Also in PS-OCT retardation and DOPU images, the mouse eye recapitulates the polarization characteristics of its human counterpart, ${ }^{56}$ while the anterior and posterior layers preserve the polarization state of backscattered light, the corneal stroma exhibits a depolarizing behavior [Figs. 4(c) and 4(d)]. Averaged axial profiles of reflectivity, retardation, and DOPU averaged over 120 A-lines are plotted in Fig. 4(f) in order to visualize the depolarizing character of the stroma via the increased spread of retardation values and the decreased DOPU values. Histological sections of the mouse cornea are shown for comparison in Figs. 4(b) and 4(e).
The depolarization characteristics of the central corneal stroma, iris, and the anterior part of the lens were evaluated in six C57BL/6 mouse eyes. In DOPU B-scan images, the average DOPU value was assessed in a manually selected region of interest spanning $150(x) \times 25(z)$ pixels $(\sim 512 \mu \mathrm{m} \times 43 \mu \mathrm{m})$ for each tissue. Box plots of the results are shown in Fig. $4(\mathrm{~g})$ for cornea, iris, and lens. The strongest depolarization was observed in the iris (DOPU $=0.63 \pm 0.02$, mean \pm standard deviation), followed by the corneal stroma (DOPU $=$ $0.79 \pm 0.03)$ and the lens (DOPU $=0.93 \pm 0.01)$. Using Mann-Whitney U-tests with Bonferroni correction, the differences between DOPU measurements were found to be statistically significant when comparing cornea versus iris $(p<0.01)$, lens versus iris $(p<0.01)$, and between corneal stroma and lens $(p<0.05)$.

\subsection{PS-OCT of Mydriasis}

In order to investigate the effect of mydriasis on the mouse eye, longitudinal imaging was performed before and after applying dilating eye drops (phenylephrine, $2.5 \%$ ) on the cornea of an anaesthetized mouse. No artificial tear drops were used in this experiment. Seventeen 3-D PS-OCT data sets were acquired between 24 and 57 min after inducing anesthesia. Volume renderings of the reflectivity data sets are shown in Figs. 5(a) and 5(d) for the undilated eye and 33 min after application of the mydriatic agent. Corresponding reflectivity and DOPU B-scans are shown in Figs. 5(b), 5(e) and 5(c), 5(f), respectively. Aside from the pupil dilation, spontaneous cataract formation can be observed as an increase of backscattering from the lens. Note that most of the lens appears polarization preserving while only a small fraction at the right-hand side of panel (f) exhibits decreased DOPU. The pupil area was assessed automatically from a depth projection image of the volumetric DOPU data and is plotted longitudinally in Fig. 5(g). An almost linear increase by $150 \%$ can be observed over a time span of 10 min following phenylephrine application; then a plateau leveling off at $\sim 2.5 \mathrm{~mm}^{2}$ is reached. In order to test the hypothesis whether depolarization in the iris increases as the iris is compacted during mydriasis, the iris was segmented and histograms of DOPU within the iris were computed for every 3-D PS-OCT data set. The results are plotted as a function of time in Fig. 5(h). Although a slight modulation of the shape of the histograms can be observed over time, its maximum essentially stays constant at $\mathrm{DOPU} \sim 0.6$.

\subsection{PS-OCT of Corneal Pathology}

PS-OCT imaging was performed in the eye of a C57BL/6 mouse, which had spontaneously developed corneal pathology, possibly scarring following a scratch. Figures 6(a)-6(c) show volume renderings of 3-D PS-OCT images. The cornea-a smooth, thin, and almost transparent structure in healthy mouse eyes-presents with a rough surface, substantial thickening, and pronounced backscattering in the reflectivity image [Fig. 6(a)]. With $192 \mu \mathrm{m}$, the central corneal thickness of this mouse eye was substantially greater than those of healthy littermates ( $72 \pm 8 \mu \mathrm{m}$, average of $N=6$ eyes). Aside from several streak-like regions of increased retardation and low DOPU, the retardation and DOPU images in Figs. 6(b) and 6(c) depict rather uniform polarization states in the cornea. With an average DOPU value of 0.94 in this cornea, depolarization was less pronounced than in the stroma of normal corneas where an average 

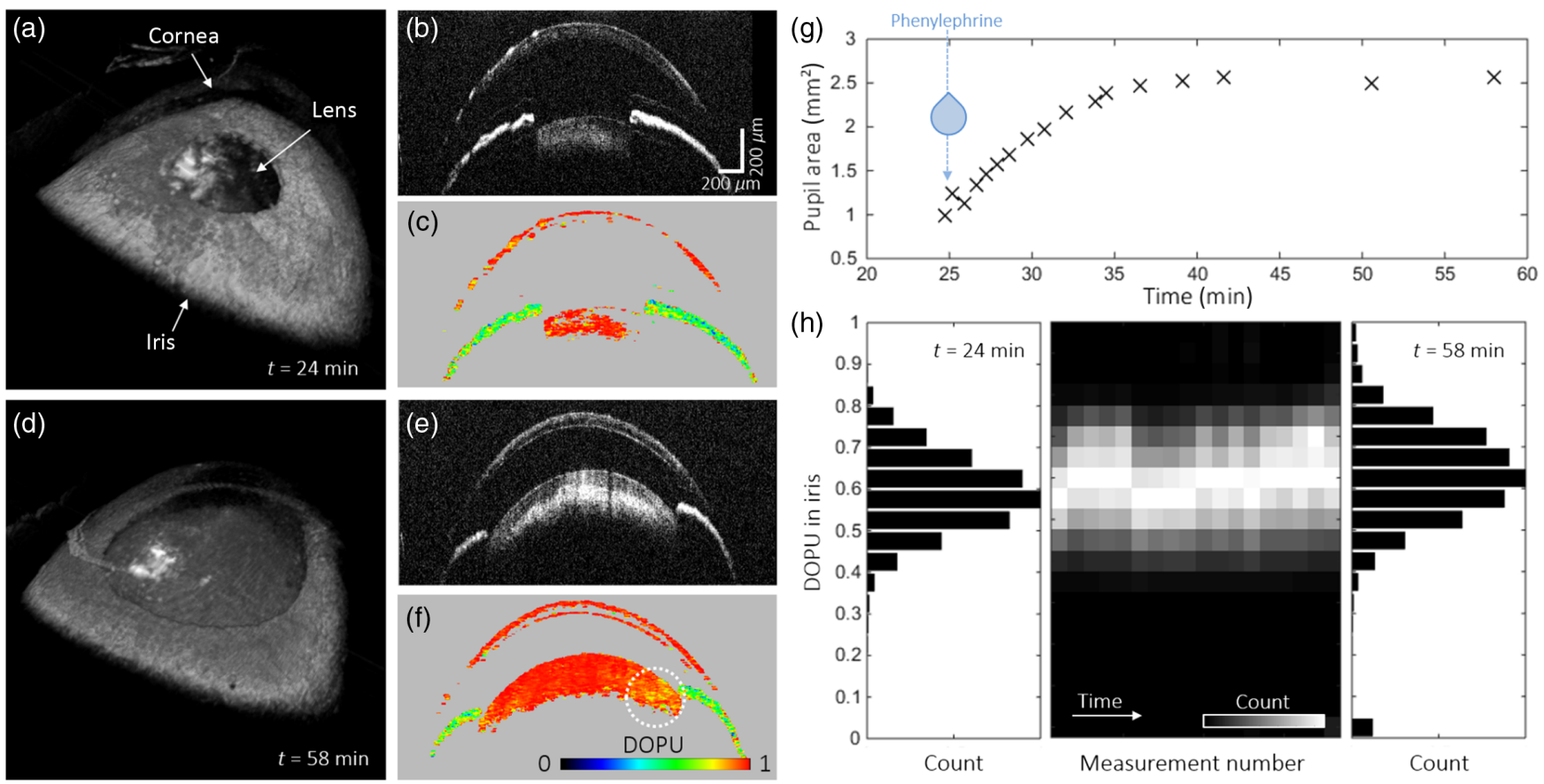

Fig. 5 Longitudinal imaging of mydriasis by phenylephrine. (a)-(c) Images of the mouse anterior segment after $24 \mathrm{~min}$ of anaesthesia and right before application of the dilating drug. A small pupil size and a clear lens can be observed. (d)-(f) PS-OCT images at $58 \mathrm{~min}$. The pupil area has more than doubled. Cataract formation can be observed. (a) and (d) Reflectivity volume renderings, (b) and (e) central reflectivity cross section, (c) and (f) corresponding DOPU image showing pronounced polarization scrambling in the iris. Note an area with decreased DOPU within the lens marked by a dotted circle. The DOPU color map in panel (f) also applies to panel (c). Scale bars in (b) also apply to (c), (e), and (f). (g) Time course of pupil area during mydriasis. Every cross corresponds to the pupil area assessment in one PS-OCT volume. (h) Histograms of DOPU in the iris during mydriasis are shown for the first and last data set on the left and right, respectively. DOPU histograms for all data sets are plotted on a gray scale normalized to the maximum count.

DOPU of $0.79 \pm 0.03$ was measured. Similar to healthy eyes, strong depolarization can be observed in the iris as well as in the pigmented hairs and eye lashes. Compared to the volume renderings, which provide a plastic overview but integrate the PS-OCT signal along the direction of observation, a fairly different picture of the cornea is seen in the PS-OCT B-scans presented in Figs. 6(d)-6(m). Unlike in normal eyes, strong birefringence can be observed in the stroma of the swollen cornea, visible as a gradual increase of phase retardation with depth [Figs. 6(f) and 6(g)].

Corneal PS-OCT projection images of the same data set computed by averaging the signals in beam direction for a shallow tissue slab are shown in Fig. 7. The mean intensity projection image averaged over the image range in Fig. 7(a) reveals a highly scattering region in the center of the cornea. Abnormal corneal swelling and hyperscattering behavior is visualized in a side-by-side comparison with a reflectivity Bscan of a normal mouse cornea in Fig. 7(d). A phase retardation map of a $17-\mu \mathrm{m}$ deep slab located $\sim 60 \mu \mathrm{m}$ posterior to the surface of the corneal epithelium is shown in Fig. 7(b). Several regions exhibiting increased retardation as well as three highly birefringent streaks can be observed. The birefringent axis orientation map of the same slab reveals partially organized birefringent fiber orientations [Fig. 7(c)]. The retardation and axis orientation B-scans show a polarization preserving characteristic in the anterior cornea, whereas weak birefringence and defined axis orientations can be observed in the portion posterior to the epithelium [Figs. 7(e) and 7(f)]. Also a highly birefringent structure can be observed, which casts a shadow trail of increased retardation [in green and marked by the arrow in Fig. 7(e)].

\section{Discussion}

The benefit of polarization contrast in OCT has been demonstrated in the literature for various diseases of the human eye. ${ }^{3}$ In disease models of human retinal pathology, PS-OCT has also proven a valuable contrast enhancement and an additional route toward the quantitative assessment of tissue parameters and lesion properties. ${ }^{39,40,57}$ Although PS-OCT has been successfully demonstrated for imaging the human anterior eye in health and disease by multiple experiments and studies, this is - to the best of our knowledge - the first presentation of PS-OCT in the anterior segment of murine eyes.

In analogy to the application of PS-OCT in human retinas and mouse models of retinal diseases, polarization provides access to additional contrast for preclinical imaging of the anterior eye. The mouse eye shares many similarities with the human eye. However, the ocular proportions differ between the two species: most apparently, the murine eye features a much larger lens compared to the overall eye size. Still, the different anatomical structures and tissue properties widely resemble those in humans. Also the polarization characteristics observed in the mouse eye (Figs. 3 and 4) and in human eyes ${ }^{12,56,58}$ are largely similar: the cornea features polarization preserving epithelial and endothelial layers, whereas light backscattered from the 


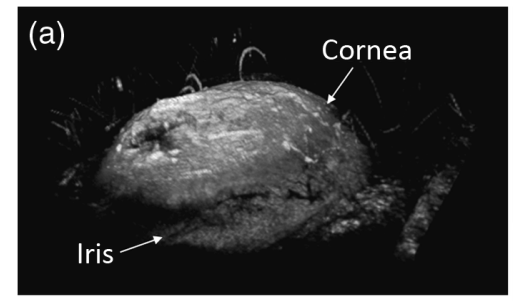

(b)

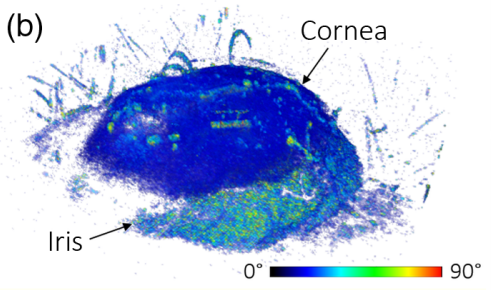

(c)

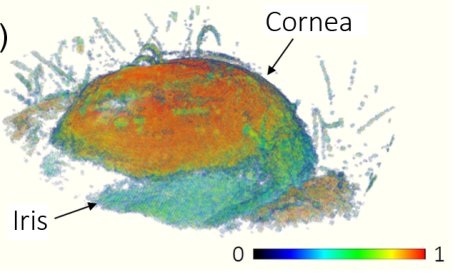

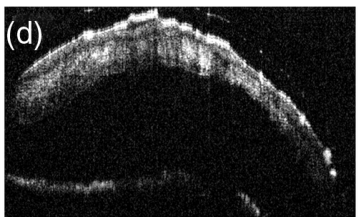
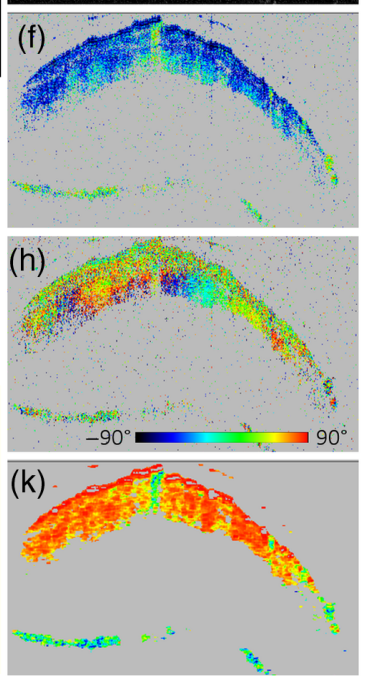
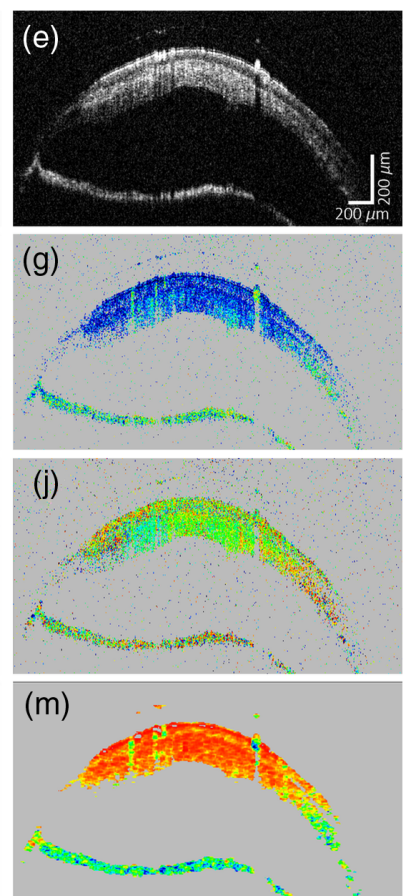

Fig. 6 PS-OCT of pathologic mouse cornea presenting a rough surface and severe thickening. Volume renderings and representative B-scans are shown in $(a)-(c)$ and $(d)-(m)$, respectively. Unlike in normal eyes, the cornea appears swollen and within the stroma strong birefringence can be observed. (a), (d), and (e) reflectivity; (b), (f), and (g) retardation; $(\mathrm{h})$ and (j) axis orientation; and (c), (k), and (m) DOPU. See panels (b), (c), and (h) for the respective PS-OCT color maps of retardation, DOPU, and axis orientation, respectively.

stroma exhibits strong local differences in the detected polarization states (Fig. 4). A different polarization pattern was observed in the pathologic cornea shown in Figs. 6 and 7. Other than the healthy case in Figs. 3 and 4, the swollen tissue enabled the visualization of the birefringent characteristics of the stroma, which may indicate altered tissue microstructure caused by an infection or scarring.

The murine lens mostly maintained the polarization state in our PS-OCT data. When severe cataracts formed under anesthesia, the lens visually became opaque and exhibited gradually increased backscattering. Also some small areas with reduced DOPU were observed in the lens, which otherwise did not markedly differ in terms of reflectivity (see Fig. 5). As anesthesia induced cataracts are reversible, no histological examination to explore the cause of the depolarizing signals was performed for the mouse eye imaged here. Nonetheless, PS-OCT may be an interesting modality to image cataractous lenses in mouse models of age-related and/or congenital lens pathology with additional contrast.

Melanin pigmentation in anterior eye tissues depolarizes backscattered light and thus can be detected by PS-OCT. Polarization scrambling was observed in the ciliary body and the iris, which may be considered the extensions of the pigmented uvea from the posterior eye's choroid. However, unlike the human iris, whose backside is covered by a single-cell layer of epithelial pigment cells, we observed depolarization in PSOCT images and accordingly melanin granules in histologic sections all across the murine iris. As PS-OCT can be used to quantify melanin density via depolarization, ${ }^{49,59}$ we also assessed DOPU in the iris during drug-induced pupil dilation. In our longitudinal PS-OCT imaging experiment, however, we could not observe any marked changes of iridal depolarization. While iris contraction may not suffice to distinctly alter pigment density within the tissue, PS-OCT powered depolarization imaging may be a potent tool for noninvasively assessing pigmented uveal tumors in a transgenic mouse model ${ }^{60}$ Also in the popular DBA/2J mouse model, PS-OCT could be useful to image iris atrophy and pigment dispersion. ${ }^{61}$

Quantitative DOPU measurements in cornea, lens, and iris revealed statistically significant differences between the three tissues [see Fig. 4(g)], which may be exploited for automated segmentation of these structures. Compared to the DOPU values measured in normal mouse corneas, corneal depolarization was less pronounced in the pathologic cornea shown in Fig. 6. This cornea was also substantially thicker than normal corneas. Although the central corneal thicknesses of $72 \pm 8 \mu \mathrm{m}$ measured with our PS-OCT system in normal C57BL/6 mice were much thinner than those published in a histological report $(137.2 \pm 14.0 \mu \mathrm{m}),{ }^{62}$ only slightly greater thicknesses $(90.8 \pm 4.6 \mu \mathrm{m})$ have been observed using OCT at $1310 \mathrm{~nm} .^{63}$ The quite substantial difference between corneal thickness measured using OCT and histology, respectively, may be attributed to morphological changes caused by tissue processing. This is also supported by a comparative study published by Hanlon et al. who compared corneal thickness measurements by OCT and conventional histology and found 30\% to $35 \%$ greater thickness for standard histological workup. ${ }^{64}$

There are some technological limitations to the PS-OCT approach presented in this paper. A spectral domain PS-OCT prototype operating in the 840-nm regime was used for imaging. With standard OCT resolution of $5.6 \mu \mathrm{m}$ in tissue $(n=1.35)$, the system was able to resolve all major structures in the anterior 

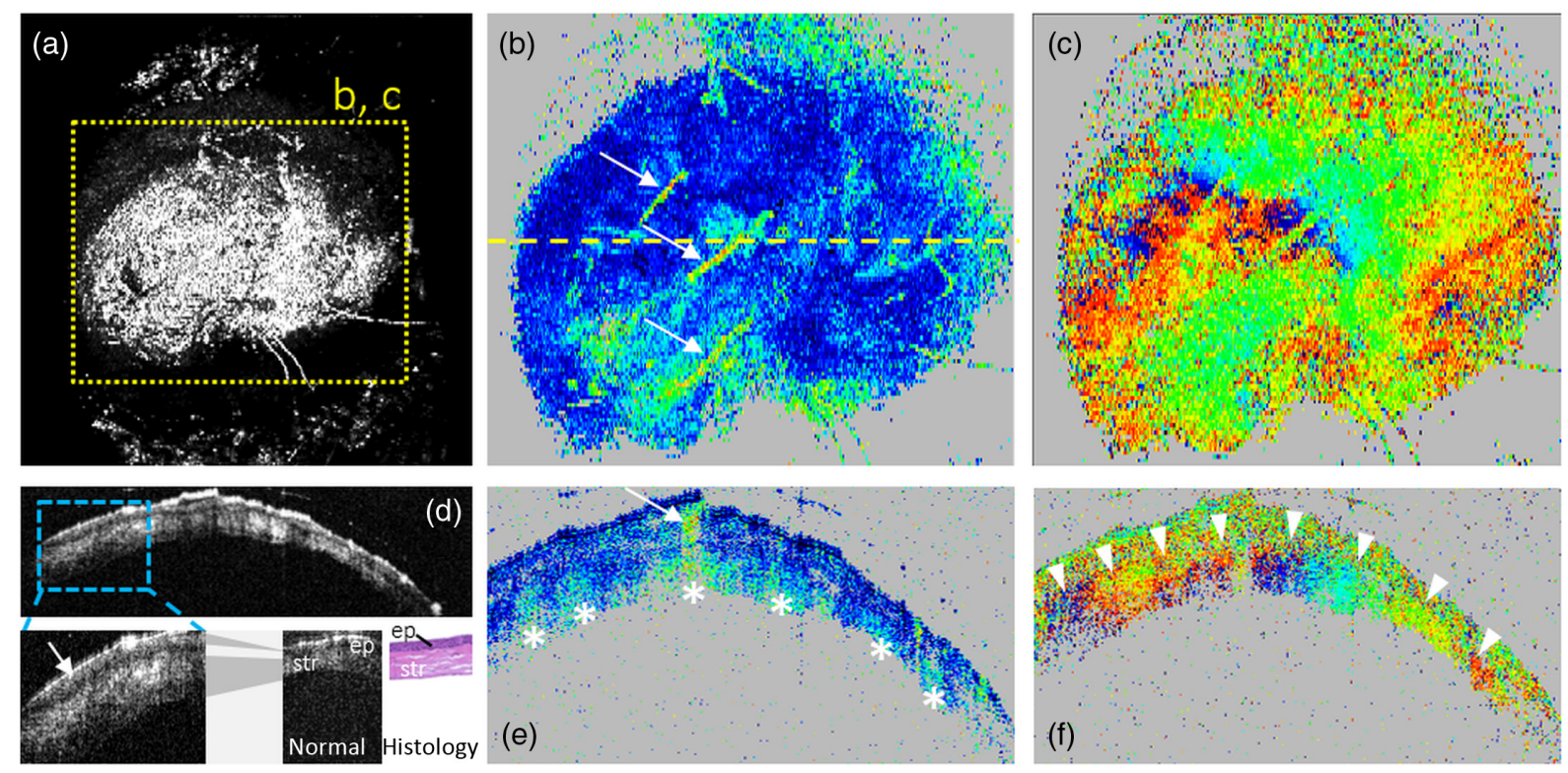

Fig. 7 PS-OCT projection maps of pathologic mouse cornea. (a) Mean intensity projection image revealing a highly scattering region in the center of the cornea. (b) Map of phase retardation averaged axially in a $17-\mu \mathrm{m}$ deep slab located $\sim 60 \mu \mathrm{m}$ posterior to the anterior surface of the cornea. Aside from several regions exhibiting increased retardation (greenish), three highly birefringent streaks can be observed (indicated by white arrows). (c) Axis orientation map of the same slab revealing organized birefringent fiber orientations. (d) Reflectivity B-scan at the location indicated by the yellow dashed line in (b). The zoom-in on the bottom left shows more than doubled corneal thickness compared to a representative healthy cornea on the bottom right. In the pathologic cornea, aside from the hyperscattering stroma (str) and the hyposcattering epithelium (ep) also an additional hyperscattering structure can be observed in the epithelium (white arrow). (e) Phase retardation B-scan image: the anterior part of the cornea appears polarization preserving, whereas postepithelial tissue marked by asterisks shows a mildly birefringent characteristic. A highly birefringent structure-potentially scar tissue resulting from a corneal scratch - can be observed close to the apex (indicated by an arrow). (f) Axis orientation B-scan image: defined birefringent axis orientations can be observed in the posterior part of the cornea. The anterior border of this ordered birefringent slab is located close to the posterior edge of the epithelium and marked by arrow heads. See Fig. 6 for the respective PS-OCT color maps.

mouse eye. In order to resolve more subtle cellular features and to decrease speckle size for improved depolarization imaging, higher axial resolution would be required. ${ }^{65,66}$ Using a light source with broader bandwidth, OCT reflectivity imaging of the anterior segment with ultrahigh axial resolution has been presented in the literature and recently enabled the visualization of single corneal nerves and the limbal palisades of Vogt. ${ }^{55,67}$ Ultrahigh resolution PS-OCT imaging has been demonstrated in the human cornea ${ }^{68}$ and may facilitate the identification of small structures such as corneal lamellae or ciliary processes in the mouse eye.

Another limitation is the rather shallow image depth that can be utilized for covering anterior eye structures. Despite the prototype's ranging depth of $2.7 \mathrm{~mm}$ in tissue, which in general would be more than sufficient to image the murine anterior segment, the shallow depth of field and the strong sensitivity rolloff limit the useful image depth to $\sim 0.5 \mathrm{~mm}$. Moreover, the cornea has distinct directional scattering properties, which reduce the signal for nonorthogonal beam-cornea geometries. The latter drawback could be overcome by implementing a nontelecentric scanning approach. ${ }^{56}$ In order to increase the utilizable image depth, PS-OCT based on swept source OCT can provide a shallower sensitivity roll-off and deeper light penetration through cataract can be achieved by using a longer-wavelength light source. ${ }^{69}$ Also the depth of field could be increased by using Bessel beams. ${ }^{70}$ Swept source PS-OCT may then enable imaging of the entire anterior chamber and lens, and even whole eye imaging in rodents. ${ }^{71,72}$ Another strategy to achieve high lateral resolution over a long depth range would be the fusion of several 3-D data sets acquired at different focus positions, which could be enabled by rapidly tunable optics as recently proposed by Grulkowski et al. ${ }^{73}$ Finally, in addition to PS-OCT imaging of mouse models, it would be particularly interesting to investigate ocular structures in rats, which have larger eyes and may allow studies of further experimental models for diseases such as glaucoma. ${ }^{29}$

In conclusion, we have demonstrated the use of PS-OCT for imaging the murine anterior segment. Our results revealed anterior eye tissues with polarization properties similar to their human counterparts. Given the wide variety of mouse models of anterior eye pathology such as keratoconus or cataract, PS-OCT might be an interesting candidate for noninvasive, real-time 3-D imaging in longitudinal studies and experimental eye research.

\section{Disclosures}

The authors declare that there are no conflicts of interest related to this article.

\section{Acknowledgments}

The authors thank Stanislava Fialová, Erich Götzinger, Harald Sattmann, Siegfried Gollubits, Roberto Plasenzotti, and Jasmin 
Rezek at the Medical University of Vienna for excellent technical support. Financial support by the Austrian Science Fund (FWF Grant Nos. P19624-B02 and P25823-B24), the European Union (FP7 HEALTH Program Grant No. 201880, FUN-OCT), and the European Research Council (ERC Starting Grant No. 640396, OPTIMALZ) is gratefully acknowledged. This work was presented in part at ARVO Imaging in the Eye Conference 2017 and SPIE Photonics West 2018. ${ }^{74}$

\section{References}

1. J. F. de Boer, C. K. Hitzenberger, and Y. Yasuno, "Polarization sensitive optical coherence tomography-a review [Invited]," Biomed. Opt. Express 8(3), 1838-1873 (2017).

2. B. Baumann, "Polarization sensitive optical coherence tomography: a review of technology and applications," Appl. Sci. 7(5), 474 (2017).

3. M. Pircher, C. K. Hitzenberger, and U. Schmidt-Erfurth, "Polarization sensitive optical coherence tomography in the human eye," Prog. Retinal Eye Res. 30(6), 431-451 (2011).

4. B. Cense et al., "Thickness and birefringence of healthy retinal nerve fiber layer tissue measured with polarization-sensitive optical coherence tomography," Invest. Ophthalmol. Visual Sci. 45(8), 2606-2612 (2004).

5. M. Pircher et al., "Imaging of polarization properties of human retina in vivo with phase resolved transversal PS-OCT," Opt. Express 12(24), 5940-5951 (2004).

6. E. Götzinger, M. Pircher, and C. K. Hitzenberger, "High speed spectral domain polarization sensitive optical coherence tomography of the human retina," Opt. Express 13(25), 10217-10229 (2005).

7. B. Baumann et al., "Single camera based spectral domain polarization sensitive optical coherence tomography," Opt. Express 15(3), 10541063 (2007).

8. M. Yamanari et al., "Phase retardation measurement of retinal nerve fiber layer by polarization-sensitive spectral-domain optical coherence tomography and scanning laser polarimetry," J. Biomed. Opt. 13(1), 014013 (2008).

9. E. Götzinger et al., "Imaging of birefringent properties of keratoconus corneas by polarization-sensitive optical coherence tomography," Invest. Ophthalmol. Visual Sci. 48(8), 3551-3558 (2007).

10. B. Cense et al., "Retinal imaging with polarization-sensitive optical coherence tomography and adaptive optics," Opt. Express 17(24), 21634-21651 (2009).

11. M. Yamanari et al., "Visualization of phase retardation of deep posterior eye by polarization-sensitive swept-source optical coherence tomography with 1- $\mu$ m probe," Opt. Express 17(15), 12385-12396 (2009).

12. Y. Lim et al., "Birefringence measurement of cornea and anterior segment by office-based polarization-sensitive optical coherence tomography," Biomed. Opt. Express 2(8), 2392-2402 (2011).

13. M. Yamanari et al., "Scleral birefringence as measured by polarizationsensitive optical coherence tomography and ocular biometric parameters of human eyes in vivo," Biomed. Opt. Express 5(5), 1391-1402 (2014).

14. M. Miura et al., "Imaging polarimetry in age-related macular degeneration," Invest. Ophthalmol. Visual Sci. 49(6), 2661-2667 (2008).

15. A. Miyazawa et al., "Tissue discrimination in anterior eye using three optical parameters obtained by polarization sensitive optical coherence tomography," Opt. Express 17(20), 17426-17440 (2009).

16. C. Ahlers et al., "Imaging of the retinal pigment epithelium in agerelated macular degeneration using polarization-sensitive optical coherence tomography," Invest. Ophthalmol. Visual Sci. 51(4), 2149-2157 (2010).

17. B. Baumann et al., "Segmentation and quantification of retinal lesions in age-related macular degeneration using polarization-sensitive optical coherence tomography," J. Biomed. Opt. 15(6), 061704 (2010).

18. J. Lammer et al., "Detection and analysis of hard exudates by polarization-sensitive optical coherence tomography in patients with diabetic maculopathy," Invest. Ophthalmol. Visual Sci. 55(3), 1564-1571 (2014).
19. B. Braaf et al., "Fiber-based polarization-sensitive OCT of the human retina with correction of system polarization distortions," Biomed. Opt. Express 5(8), 2736-2758 (2014).

20. P. Roberts et al., "Retinal pigment epithelial features in central serous chorioretinopathy identified by polarization-sensitive optical coherence tomography," Invest. Ophthalmol. Visual Sci. 57(4), 1595-1603 (2016).

21. M. Miura et al., "Evaluation of intraretinal migration of retinal pigment epithelial cells in age-related macular degeneration using polarimetric imaging," Sci. Rep. 7(1), 3150 (2017).

22. S. Fukuda et al., "Keratoconus diagnosis using anterior segment polarization-sensitive optical coherence tomography," Invest. Ophthalmol. Visual Sci. 54(2), 1384-1391 (2013).

23. M. J. Ju and S. Tang, "Usage of polarization-sensitive optical coherence tomography for investigation of collagen cross-linking," J. Biomed. Opt. 20(4), 046001 (2015).

24. Y. Yasuno et al., "Investigation of post-glaucoma-surgery structures by three-dimensional and polarization sensitive anterior eye segment optical coherence tomography," Opt. Express 17(5), 3980-3996 (2009).

25. S. Fukuda et al., "Noninvasive evaluation of phase retardation in blebs after glaucoma surgery using anterior segment polarization-sensitive optical coherence tomography," Invest. Ophthalmol. Visual Sci. 55(8), 5200-5206 (2014).

26. D. Kasaragod et al., "Objective evaluation of functionality of filtering bleb based on polarization-sensitive optical coherence tomography," Invest. Ophthalmol. Visual Sci. 57(4), 2305-2310 (2016).

27. S. Fukuda et al., "Comparison of intensity, phase retardation, and local birefringence images for filtering blebs using polarization-sensitive optical coherence tomography," Sci. Rep. 8(1), 7519 (2018).

28. G. J. Chader, "Animal models in research on retinal degenerations: past progress and future hope," Vision Res. 42(4), 393-399 (2002).

29. T. V. Johnson and S. I. Tomarev, "Rodent models of glaucoma," Brain Res. Bull. 81(2), 349-358 (2010).

30. M. Asai-Coakwell et al., "Reduced human and Murine Corneal thickness in an Axenfeld-Rieger syndrome subtype," Invest. Ophthalmol. Visual Sci. 47(11), 4905-4909 (2006).

31. T.-H. Chou et al., "Postnatal elongation of eye size in DBA/2J mice compared with C57BL/6J mice: in vivo analysis with whole-eye OCT," Invest. Ophthalmol. Visual Sci. 52(6), 3604-3612 (2011).

32. G. Li et al., "Disease progression in iridocorneal angle tissues of BMP2induced ocular hypertensive mice with optical coherence tomography," Mol. Vision 20, 1695-1709 (2014)

33. G. Li et al., "Pilocarpine-induced dilation of Schlemm's Canal and prevention of Lumen collapse at elevated intraocular pressures in living mice visualized by OCT," Invest. Ophthalmol. Visual Sci. 55(6), 3737-3746 (2014).

34. H. Yun et al., "A laser-induced mouse model with long-term intraocular pressure elevation," PLoS One 9(9), e107446 (2014).

35. H. Ho et al., "Altered anterior segment biometric parameters in mice deficient in SPARC," Invest. Ophthalmol. Visual Sci. 58(1), 386-393 (2017).

36. D. R. Koehn, K. J. Meyer, and M. G. Anderson, "Genetic evidence for differential regulation of corneal epithelial and stromal thickness," Invest. Ophthalmol. Visual Sci. 56(9), 5599-5607 (2015).

37. B. Baumann et al., "Peripapillary rat sclera investigated in vivo with polarization-sensitive optical coherence tomography," Invest. Ophthalmol. Visual Sci. 55(11), 7686-7696 (2014).

38. S. Fialová et al., "Polarization properties of single layers in the posterior eyes of mice and rats investigated using high resolution polarization sensitive optical coherence tomography," Biomed. Opt. Express 7(4), 1479-1495 (2016).

39. M. Augustin et al., "Multi-functional OCT enables longitudinal study of retinal changes in a VLDLR knockout mouse model," PLoS One 11(10), e0164419 (2016).

40. S. Fialová et al., "Posterior rat eye during acute intraocular pressure elevation studied using polarization sensitive optical coherence tomography," Biomed. Opt. Express 8(1), 298-314 (2017).

41. E. Götzinger et al., "Speckle noise reduction in high speed polarization sensitive spectral domain optical coherence tomography," Opt. Express 19(15), 14568-14585 (2011). 
42. M. Wojtkowski et al., "Ultrahigh-resolution, high-speed, Fourier domain optical coherence tomography and methods for dispersion compensation," Opt. Express 12(11), 2404-2422 (2004).

43. B. Baumann et al., "Visualization of neuritic plaques in Alzheimer's disease by polarization-sensitive optical coherence microscopy," Sci. Rep. 7, 43477 (2017)

44. R. Tripathi et al., "Spectral shaping for non-Gaussian source spectra in optical coherence tomography," Opt. Lett. 27(6), 406-408 (2002).

45. E. Gotzinger et al., "Retinal pigment epithelium segmentation by polarization sensitive optical coherence tomography," Opt. Express 16(21), 16410-16422 (2008).

46. S. Makita et al., "Degree of polarization uniformity with high noise immunity using polarization-sensitive optical coherence tomography," Opt. Lett. 39(24), 6783-6786 (2014).

47. J. Schindelin et al., "Fiji: an open-source platform for biological-image analysis," Nat. Methods 9, 676-682 (2012).

48. M. J. Ju et al., "Advanced multi-contrast Jones matrix optical coherence tomography for Doppler and polarization sensitive imaging," Opt. Express 21(16), 19412-19436 (2013).

49. B. Baumann et al., "Melanin pigmentation in rat eyes: in vivo imaging by polarization-sensitive optical coherence tomography and comparison to histology," Invest. Ophthalmol. Visual Sci. 56(12), 7462-7472 (2015).

50. N. Lippok et al., "Quantitative depolarization measurements for fiberbased polarization-sensitive optical frequency domain imaging of the retinal pigment epithelium," J. Biophotonics e201800156 (2018).

51. M. Bonesi et al., "High-speed polarization sensitive optical coherence tomography scan engine based on Fourier domain mode locked laser," Biomed. Opt. Express 3(11), 2987-3000 (2012).

52. M. Sugita et al., "Analysis of optimum conditions of depolarization imaging by polarization-sensitive optical coherence tomography in the human retina," J. Biomed. Opt. 20(1), 016011 (2015).

53. E. Götzinger et al., "Three-dimensional polarization sensitive OCT imaging and interactive display of the human retina," Opt. Express 17(5), 4151-4165 (2009).

54. N. S. Wolf et al., "Normal mouse and rat strains as models for agerelated cataract and the effect of caloric restriction on its development," Exp. Eye Res. 70(5), 683-692 (2000).

55. R. M. Werkmeister et al., "Ultrahigh-resolution OCT imaging of the human cornea," Biomed. Opt. Express 8(2), 1221-1239 (2017).

56. F. Beer et al., "Conical scan pattern for enhanced visualization of the human cornea using polarization-sensitive OCT," Biomed. Opt. Express 8(6), 2906-2923 (2017).

57. M. Augustin et al., "In vivo characterization of spontaneous retinal neovascularization in the mouse eye by multifunctional optical coherence tomography," Invest. Ophthalmol. Visual Sci. 59(5), 2054-2068 (2018).

58. E. Götzinger et al., "Measurement and imaging of birefringent properties of the human cornea with phase-resolved, polarization-sensitive optical coherence tomography," J. Biomed. Opt. 9(1), 9 (2004).

59. B. Baumann et al., "Polarization sensitive optical coherence tomography of melanin provides intrinsic contrast based on depolarization," Biomed. Opt. Express 3(7), 1670-1683 (2012).

60. T. R. Kramer et al., "Pigmented uveal tumours in a transgenic mouse model," Br. J. Ophthalmol. 82(8), 953-960 (1998).

61. S. W. John et al., "Essential iris atrophy, pigment dispersion, and glaucoma in DBA/2J mice," Invest. Ophthalmol. Visual Sci. 39(6), 951-962 (1998)

62. J. T. Henriksson, A. M. McDermott, and J. P. G. Bergmanson, "Dimensions and morphology of the cornea in three strains of mice," Invest. Ophthalmol. Visual Sci. 50(8), 3648-3654 (2009).

63. X. Zhou et al., "Biometric measurement of the mouse eye using optical coherence tomography with focal plane advancement," Vision Res. 48(9), 1137-1143 (2008).

64. S. D. Hanlon, N. B. Patel, and A. R. Burns, "Assessment of postnatal corneal development in the C57BL/6 mouse using spectral domain optical coherence tomography and microwave-assisted histology," Exp. Eye Res. 93(4), 363-370 (2011)

65. E. Götzinger et al., "Polarization maintaining fiber based ultra-high resolution spectral domain polarization sensitive optical coherence tomography," Opt. Express 17(25), 22704-22717 (2009).
66. B. Cense, M. Reddikumar, and J. Cervantes, "Ultra-high resolution polarization-sensitive optical coherence tomography for imaging of the retinal nerve fiber layer," Proc. SPIE 10251, 102510S (2017).

67. K. Bizheva et al., "Sub-micrometer axial resolution OCT for in-vivo imaging of the cellular structure of healthy and keratoconic human corneas," Biomed. Opt. Express 8(2), 800-812 (2017).

68. M. Pircher et al., "Ultrahigh-resolution polarization-sensitive optical coherence tomography," Proc. SPIE 5690, 6 (2005).

69. B. Povazay et al., "Wide-field optical coherence tomography of the choroid in vivo," Invest. Ophthalmol. Visual Sci. 50(4), 1856-1863 (2009).

70. R. A. Leitgeb et al., "Extended focus depth for Fourier domain optical coherence microscopy," Opt. Lett. 31(16), 2450-2452 (2006).

71. L. Wang et al., "Heritability of ocular component dimensions in mice phenotyped using depth-enhanced swept source optical coherence tomography," Exp. Eye Res. 93(4), 482-490 (2011).

72. J. J. Liu et al., "In vivo imaging of the rodent eye with swept source/Fourier domain OCT," Biomed. Opt. Express 4(2), 351-363 (2013).

73. I. Grulkowski et al., "Swept source optical coherence tomography and tunable lens technology for comprehensive imaging and biometry of the whole eye," Optica 5(1), 52-59 (2018).

74. B. Baumann et al., "Polarization-sensitive optical coherence tomography in the anterior mouse eye (Conference Presentation)," Proc. SPIE 10474, 1047408 (2018).

Bernhard Baumann studied physics at the University of Vienna and received his $\mathrm{PhD}$ in medical physics from the Medical University of Vienna in 2009. He is an associate professor at the Center for Medical Physics and Biomedical Engineering of the Medical University of Vienna. His research interests are the development of innovative optical methods for biomedical imaging -in particular optical coherence tomography (OCT) - and their application for improved diagnostics of diseases in both clinical and preclinical research.

Marco Augustin received his master's degree in medical informatics from Vienna University of Technology in 2014 and his PhD in medical physics from the Medical University of Vienna in 2018, respectively. Currently, he is enrolled as a postdoctoral associate at the Center for Medical Physics and Biomedical Engineering of the Medical University of Vienna. His interests include optical imaging techniques, especially optical coherence tomography, and its functional extensions, as well as image processing and pattern recognition particularly in life sciences.

Antonia Lichtenegger received her master's degree in technical mathematics and biomedical engineering from the Technical University of Vienna in 2014 and 2015, respectively. Currently, she is a PhD candidate at the Center for Medical Physics and Biomedical Engineering of the Medical University of Vienna. Her interests include designing and setting up optical coherence microscopy systems and image processing in biomedical optics and neuroscience.

Danielle J. Harper received her master's degree in physics at the University of St. Andrews in the United Kingdom in 2015. As a PhD student at the Medical University of Vienna, she is currently working to design an innovative OCT system, which is tailored for the in vivo functional imaging of the retina of animal models. Her main interests lie in optical imaging, particularly in the optical designs that make techniques such as OCT possible.

Martina Muck received her BSc degree in biomedical science and her master's degree in tissue engineering and regenerative medicine from the University of Applied Sciences, FH Campus Wien and Technikum in Vienna. As a biomedical analyst, she is working on the preparation of brain samples for OCM imaging and histology.

Pablo Eugui received his master's degree in biomedical engineering from the Universidad Publica de Navarra, Spain, in 2015. Currently, he is enrolled as a PhD student of the Medical University of Vienna and is working on fiber-based optical coherence tomography systems. His interests are optical imaging with different techniques, signal and image processing applied to the medical field, and the study of different neurological diseases. 
Andreas Wartak received his $\mathrm{PhD}$ in medical physics from the Medical University of Vienna, Austria, in 2018 . He is currently enrolled as a postdoctoral researcher at Wellman Center for Photomedicine, Harvard Medical School and Massachusetts General Hospital, Boston, Massachusetts, USA. His research focuses on OCT, in particular on the improvement and development of multichannel techniques for application in ophthalmology with emphasis on retinal imaging.

Michael Pircher received his PhD in technical sciences from Vienna University of Technology and completed his habilitation degree in medical physics at the Medical University of Vienna. He is an associate professor at the Center for Medical Physics and Biomedical Engineering of the Medical University of Vienna and an associate editor of Biomedical Optics Express.

Christoph K. Hitzenberger is a professor of medical physics and a vice-chair of the Center for Medical Physics and Biomedical Engineering at the Medical University of Vienna, and editor-in-chief of Biomedical Optics Express. He works in biomedical optics since 1987 and is one of the pioneers of low-coherence ocular biometry and OCT. He is a fellow of SPIE and OSA. His pioneering contributions to OCT were awarded with the Russ Prize of the National Academy of Engineering in 2017. 